\title{
Supporting Information \\ for Designer Chiral Quaternary Ammonium Bifluorides as an Efficient Catalyst for Asymmetric Nitroaldol Reaction of Silyl Nitronates with Aromatic Aldehydes
}

\author{
Takashi Ooi, Kanae Doda, and Keiji Maruoka* \\ Department of Chemistry, Graduate School of Science, Kyoro University \\ Sakyo, Kyoto 606-8502, Japan
}

Preparation of $C_{2}$-Symmetric Chiral Quaternary Ammonium Bifluoride 1.' Chiral quaternary ammonium bifluoride 1 was prepared by passage of a methanolic solution of the corresponding bromide through a column of ion exchange resin Amberlyst A-26 $\left(\mathrm{OH}^{-}\right)$to afford the corresponding quaternary ammonium hydroxide, followed by neutralizing with 2 equiv of $1 \mathrm{~N} \mathrm{HF}$ solution. The solvent was removed under reduced pressure, and the residue was co-evaporated with dichloromethane-hexane (1:1), then dried under vacuum. The requisite ammonium bifluoride was obtained quantitatively as fine powder and used for the asymmetric nitroaldol reaction without further purification. Recrystallization of these quaternary ammonium bifluorides, $\mathbf{1 a}$ and $\mathbf{1 b}$ were unsuccessful. $\mathbf{1 a}$ and $\mathbf{1 b}$ can be kept in a desiccator at room temperature for a month with gradual decrease of the catalytic activity, which, however, does not affect the enantioselectivity of the nitroaldol reaction.

$\boldsymbol{C}_{2}$-Symmetric Chiral Quaternary Ammonium Bifluoride 1a: $[\alpha]_{\mathrm{D}}{ }^{27} 78.0^{\circ}(c) 0.50$, $\left.\mathrm{CH}_{3} \mathrm{OH}\right) ;{ }^{1} \mathrm{H}$ NMR (400 MHz, $\left.\mathrm{CD}_{2} \mathrm{Cl}_{2}\right) \delta 8.39(2 \mathrm{H}, \mathrm{s}, \mathrm{Ar}-\mathrm{H}), 8.30(2 \mathrm{H}, \mathrm{s}, \mathrm{Ar}-\mathrm{H}), 8.20$ (2H, d, $J=7.9 \mathrm{~Hz}, \mathrm{Ar}-\mathrm{H}), 7.88(2 \mathrm{H}, \mathrm{d}, J=8.3 \mathrm{~Hz}, \mathrm{Ar}-\mathrm{H}), 7.70(2 \mathrm{H}, \mathrm{ddd}, J=1.2,6.7,7.9 \mathrm{~Hz}, \mathrm{Ar}-\mathrm{H})$, $7.53(2 \mathrm{H}, \mathrm{ddd}, J=1.2,6.7,8.3 \mathrm{~Hz}, \mathrm{Ar}-\mathrm{H}), 7.40(2 \mathrm{H}, \mathrm{ddd}, J=1.2,6.7,8.3 \mathrm{~Hz}, \mathrm{Ar}-\mathrm{H}), 7.31$ $(2 \mathrm{H}, \mathrm{d}, J=8.7 \mathrm{~Hz}, \mathrm{Ar}-\mathrm{H}), 7.24$ (2H, ddd, $J=1.2,6.7,8.3 \mathrm{~Hz}, \mathrm{Ar}-\mathrm{H}), 7.18(2 \mathrm{H}, \mathrm{d}, J=8.3 \mathrm{~Hz}$, Ar-H), $7.11(2 \mathrm{H}, \mathrm{d}, J=8.7 \mathrm{~Hz}, \mathrm{Ar}-\mathrm{H}), 8.40-7.10(6 \mathrm{H}, \mathrm{br}, \mathrm{Ar}-\mathrm{H}) 6.27(2 \mathrm{H}, \mathrm{d}, J=8.3 \mathrm{~Hz}, \mathrm{Ar}-$ $\mathrm{H}), 4.64\left(2 \mathrm{H}, \mathrm{d}, J=13.9 \mathrm{~Hz}, \mathrm{Ar}-\mathrm{CH}_{2}\right), 4.49\left(2 \mathrm{H}, \mathrm{d}, J=13.9 \mathrm{~Hz}, \mathrm{Ar}-\mathrm{CH}_{2}\right), 4.26(2 \mathrm{H}, \mathrm{d}, J=$ $\left.13.1 \mathrm{~Hz}, \mathrm{Ar}-\mathrm{CH}_{2}\right), 3.69\left(2 \mathrm{H}, \mathrm{d}, J=13.1 \mathrm{~Hz}, \mathrm{Ar}-\mathrm{CH}_{2}\right) ;{ }^{13} \mathrm{C} \mathrm{NMR}\left(100 \mathrm{MHz}, \mathrm{CDCl}_{3}\right.$, at $\left.50{ }^{\circ} \mathrm{C}\right)$ $\delta 142.07,139.59,136.49,136.37,133.87,133.78,133.64,133.26\left(q, J_{\text {C-F }}=36.9 \mathrm{~Hz}\right), 131.78$, $131.15,131.02$, 128.67, 128.57, 128.38, 128.19, 128.12, 127.51, 127.40, 126.92, 126.19, $124.89,124.47,122.34,122.14,122.09,62.37,57.41 ;{ }^{19} \mathrm{~F} \mathrm{NMR}\left(376 \mathrm{MHz}, \mathrm{CD}_{2} \mathrm{Cl}_{2}\right) \delta-62.80$, -152.20; IR (KBr) 3393, 1524, 1470, 1377, 1311, 1281, 1177, 1138, 1030, 897, 847, 750, 714, $681 \mathrm{~cm}^{-1}$; HRMS (ESI-TOF) calcd for $\mathrm{C}_{60} \mathrm{H}_{36} \mathrm{~F}_{12} \mathrm{~N}\left([\mathrm{M}]^{+}\right)$: 998.2651. Found: $998.2655\left([\mathrm{M}]^{+}\right)$. Upon measuring melting point, the light yellow solid started changing to dark red at $210{ }^{\circ} \mathrm{C}$ and it finally melted at $239{ }^{\circ} \mathrm{C}$. However, ${ }^{1} \mathrm{H}$ NMR analysis after the measurement showed almost total decomposition. 
$C_{2}$-Symmetric Chiral Quaternary Ammonium Bifluoride 1b: $[\alpha]_{\mathrm{D}}^{27} 62.3^{\circ}$ (c 1.00, $\left.\mathrm{CH}_{3} \mathrm{OH}\right) ;{ }^{1} \mathrm{H}$ NMR $\left(400 \mathrm{MHz}, \mathrm{CD}_{2} \mathrm{Cl}_{2}\right) \delta 8.87(2 \mathrm{H}, \mathrm{br}, \mathrm{Ar}-\mathrm{H}), 8.59(4 \mathrm{H}, \mathrm{br}, \mathrm{Ar}-\mathrm{H}), 8.54(2 \mathrm{H}$, s, Ar-H), 8.26 (2H, s, Ar-H), 8.23 (2H, d, $J=8.3 \mathrm{~Hz}, \mathrm{Ar}-\mathrm{H}), 8.12$ (2H, br, Ar-H), 8.10-7.90 $(4 \mathrm{H}, \mathrm{br}, \mathrm{Ar}-\mathrm{H}), 7.84(4 \mathrm{H}, \mathrm{d}, J=8.3 \mathrm{~Hz}, \mathrm{Ar}-\mathrm{H}), 7.73(2 \mathrm{H}, \mathrm{dd}, J=7.1,7.9 \mathrm{~Hz}, \mathrm{Ar}-\mathrm{H}), 7.63(2 \mathrm{H}$, br, Ar-H), $7.49(2 \mathrm{H}$, ddd, $J=0.4,7.1,7.9 \mathrm{~Hz}, \mathrm{Ar}-\mathrm{H}), 7.44(2 \mathrm{H}$, ddd, $J=0.4,7.1,7.9 \mathrm{~Hz}, \mathrm{Ar}-$ H), $7.35(2 \mathrm{H}, \mathrm{d}, J=8.7 \mathrm{~Hz}, \mathrm{Ar}-\mathrm{H}), 7.27(2 \mathrm{H}, \mathrm{d}, J=8.3 \mathrm{~Hz}, \mathrm{Ar}-\mathrm{H}), 7.18(2 \mathrm{H}$, ddd, $J=1.2,6.7$, $8.7 \mathrm{~Hz}$; Ar-H), $7.07(2 \mathrm{H}, \mathrm{d}, J=8.7 \mathrm{~Hz}, \mathrm{Ar}-\mathrm{H}), 6.58(2 \mathrm{H}, \mathrm{d}, J=8.7 \mathrm{~Hz}, \mathrm{Ar}-\mathrm{H}), 4.93(2 \mathrm{H}, \mathrm{d}, J=$ $\left.13.5 \mathrm{~Hz}, \mathrm{Ar}-\mathrm{CH}_{2}\right), 4.47\left(2 \mathrm{H}, \mathrm{d}, J=13.5 \mathrm{~Hz}, \mathrm{Ar}-\mathrm{CH}_{2}\right), 4.36\left(2 \mathrm{H}, \mathrm{d}, J=13.5 \mathrm{~Hz}, \mathrm{Ar}-\mathrm{CH}_{2}\right), 3.69$ $\left(2 \mathrm{H}, \mathrm{d}, J=13.5 \mathrm{~Hz}, \mathrm{Ar}-\mathrm{CH}_{2}\right) ;{ }^{13} \mathrm{C} \mathrm{NMR}\left(100 \mathrm{MHz}, \mathrm{CDCl}_{3}\right.$, at $\left.50{ }^{\circ} \mathrm{C}\right) \delta 142.25,141.97$, $141.49,139.65,137.84,136.75,133.99,133.83,133.30,132.75\left(\mathrm{q}, J_{\mathrm{C}-\mathrm{F}}=40.2 \mathrm{~Hz}\right), 131.69$, $131.18,130.34,128.60,128.49,128.24,127.91,127.82,127 . .65,127.49,127.07,126.61$, 126.44, 125.05, 124.50, 122.62, 122.09, 122.05, 121.76, 62.27, 57.59; ${ }^{19} \mathrm{~F}$ NMR (376 MHz, $\mathrm{CD}_{2} \mathrm{Cl}_{2}$ ) $\delta$-62.85, -151.16; IR (KBr) 3437, 3059, 1620, 1595, 1526, 1466, 1391, 1367, 1331, $1281,1178,1136,901,883,845,752,706,685 \mathrm{~cm}^{-1}$; HRMS (ESI-TOF) calcd for $\mathrm{C}_{88} \mathrm{H}_{48} \mathrm{~F}_{24} \mathrm{~N}$ $\left([\mathrm{M}]^{+}\right)$: 1574.3398 . Found: $1574.3388\left([\mathrm{M}]^{+}\right)$. Melted at $241{ }^{\circ} \mathrm{C}$ with decomposition.

General Procedure for the Preparation of Silyl Nitronate 2. ${ }^{2}$ To a stirred solution of diisopropylamine $(7.1 \mathrm{~mL}, 51 \mathrm{mmol})$ in THF $(100 \mathrm{~mL})$ was added a $1.5 \mathrm{M}$ hexane solution of $n$-BuLi $(33 \mathrm{~mL}, 50 \mathrm{mmol})$ at $0{ }^{\circ} \mathrm{C}$ under argon atmosphere. After stirring for $30 \mathrm{~min}$, this solution of lithium diisopropylamide was quickly transferred via cannular to the solution of nitroalkane $(50 \mathrm{mmol})$ in THF $(30 \mathrm{~mL})$ at $-78{ }^{\circ} \mathrm{C}$. The resulting jelly was kept at $-78{ }^{\circ} \mathrm{C}$ for $15 \mathrm{~min}$ and then trimethylsilyl chloride $(6.2 \mathrm{~mL}, 50 \mathrm{mmol})$ was added. After stirring for 15 min at the temperature, cooling bath was removed and stirring was continued for additional 2 h. The resulting mixture was evaporated and the residual semi-solid was suspended in pentane $(200 \mathrm{~mL})$, which was filtered through a Celite pad on a glass filter followed by rapid washing with pentane $(20 \mathrm{~mL} \times 2)$. Fractional distillation of the residue gave the corresponding pure silyl nitronate in $50-80 \%$ yield.

Silyl Nitronate 2d: ${ }^{1} \mathrm{H}$ NMR (400 MHz, $\left.\mathrm{CD}_{2} \mathrm{Cl}_{2}\right) \delta$ 7.31-7.19 $(5 \mathrm{H}, \mathrm{m}, \mathrm{Ph}), 6.12(1 \mathrm{H}, \mathrm{t}, J=$ $6.1 \mathrm{~Hz}, \mathrm{~N}=\mathrm{CH}), 4.43\left(2 \mathrm{H}, \mathrm{s}, \mathrm{PhCH}_{2}\right), 3.53\left(2 \mathrm{H}, \mathrm{t}, J=6.1 \mathrm{~Hz}, \mathrm{OCH}_{2} \mathrm{CH}_{2}\right), 2.49(2 \mathrm{H}, \mathrm{dt}, J=$ 6.1, 6.1 Hz, $\left.\mathrm{OCH}_{2} \mathrm{CH}_{2}\right), 0.23\left(9 \mathrm{H}, \mathrm{s}, \mathrm{SiCH}_{3}\right) ;{ }^{13} \mathrm{C} \mathrm{NMR}\left(100 \mathrm{MHz}, \mathrm{CD}_{2} \mathrm{Cl}_{2}\right)$ 138.58, 128.54 , 127.86, 127.80, 114.57, 73.19, 66.55, 27.88, 0.08; b.p. $112{ }^{\circ} \mathrm{C} / 0.003 \mathrm{mmHg}$.

General Procedure for Asymmetric Nitroaldol Reaction Catalyzed by 1b. To a solution of chiral quaternary ammonium bifluoride $\mathbf{1 b}(9.6 \mathrm{mg}, 0.006 \mathrm{mmol})$ in distilled THF ( $3 \mathrm{~mL})$ was added aldehyde $(0.3 \mathrm{mmol})$ at room temperature and the mixture was cooled to $-98{ }^{\circ} \mathrm{C}$ with methanol-liq. $\mathrm{N}_{2}$ bath under argon atmosphere. Then, a solution of silyl nitronate $\mathbf{2}(0.36$ mmol) in distilled THF $(1 \mathrm{~mL})$ was introduced over a period of $5 \mathrm{~min}$. The mixture was maintained at $-98{ }^{\circ} \mathrm{C}$ for $1 \mathrm{~h}$ and the stirring was continued at $-78{ }^{\circ} \mathrm{C}$ for an additional $1-5 \mathrm{~h}$. After the addition of $1 \mathrm{~N} \mathrm{HCl}$ at $-78^{\circ} \mathrm{C}$, it was warmed to $0{ }^{\circ} \mathrm{C}$ followed by vigorous stirring 
for 20 min and the whole mixture was extracted with ether. The combined organic extracts were washed with water and brine, and dried over $\mathrm{Na}_{2} \mathrm{SO}_{4}$. The solvent was evaporated and the residual oil was purified by column chromatography on silica gel (ether/hexane as eluant) to afford the corresponding nitroaldol adduct 3. ${ }^{3}$ The optical purities of the nitroaldol adducts were determined by HPLC analysis using DAICEL Chiralpak AS. Table 2 shows the retention times of anti isomers.

Table 2. The Retention Time of anti Isomer

\begin{tabular}{|l|c|c|c|c|}
\hline \multirow{2}{*}{ nitroaldol adduct } & \multirow{2}{*}{$\begin{array}{c}\text { eluants } \\
\text { (hexane/alcohol })\end{array}$} & $\begin{array}{c}\text { flow rate } \\
(\mathrm{mL} / \mathrm{min})\end{array}$ & \multicolumn{2}{|c|}{ retention time (min) } \\
\cline { 4 - 5 } & $50: 1(i-\mathrm{PrOH})$ & 1.0 & $26.2(1 S, 2 R)$ & $32.2(1 R, 2 S)$ \\
\hline $\mathbf{3 a}\left(\mathrm{R}^{1}=\mathrm{Me}, \mathrm{R}^{2}=\mathrm{Ph}\right)$ & $30: 1(\mathrm{EtOH})$ & 1.0 & 11.2 & 12.5 \\
3b $\left(\mathrm{R}^{1}=\mathrm{Me}, \mathrm{R}^{2}=p-\mathrm{Me}-\mathrm{Ph}\right)$ & $30: 1(\mathrm{EtOH})$ & 1.0 & 16.9 & 18.3 \\
3c $\left(\mathrm{R}^{1}=\mathrm{Me}, \mathrm{R}^{2}=p-\mathrm{F}-\mathrm{Ph}\right)$ & $30: 1(\mathrm{EtOH})$ & 1.0 & 18.1 & 19.9 \\
3d $\left(\mathrm{R}^{1}=\mathrm{Me}, \mathrm{R}^{2}=\beta-\mathrm{Np}\right)$ & $50: 1(i-\mathrm{PrOH})$ & 1.0 & 20.0 & 23.0 \\
3e $\left(\mathrm{R}^{1}=\mathrm{Et}, \mathrm{R}^{2}=\mathrm{Ph}\right)$ & $10: 1(i-\mathrm{PrOH})$ & 0.5 & 27.2 & 29.7 \\
3f $\left[\mathrm{R}^{1}=\mathrm{BnO}\left(\mathrm{CH}_{2}\right)_{2}, \mathrm{R}^{2}=\mathrm{Ph}\right]$ & & &
\end{tabular}

\section{Characterization of the Nitroaldols.}

4-Benzyloxy-2-nitro-1-phenylbutan-1-ol (3f): ${ }^{1} \mathrm{H}$ NMR (400 $\left.\mathrm{MHz}, \mathrm{CDCl}_{3}\right) \delta$ 7.37-7.21 $(10 \mathrm{H}, \mathrm{m}, \mathrm{Ph}), 5.20(0.83 \mathrm{H}, \mathrm{dd}, J=3.6,4.7 \mathrm{~Hz}, \mathrm{CHOH}$, anti isomer $), 5.00-4.94(0.34 \mathrm{H}, \mathrm{m}$, $\mathrm{CHOH}$ and $\mathrm{CHNO}_{2}$, syn isomer), $4.88\left(0.83 \mathrm{H}\right.$, ddd, $J=3.6,4.7,5.1 \mathrm{~Hz}, \mathrm{CHNO}_{2}$, anti isomer), $4.38\left(1.66 \mathrm{H}, \mathrm{s}, \mathrm{PhCH}_{2}\right.$, anti isomer $), 4.36\left(0.34 \mathrm{H}, \mathrm{s}, \mathrm{PhCH}_{2}\right.$, syn isomer $), 3.52(0.83 \mathrm{H}, \mathrm{m}$, $\mathrm{OCHCH}_{2}$, anti isomer), $3.42\left(0.83 \mathrm{H}\right.$, ddd, $J=4.4,8.7,10.3 \mathrm{~Hz}, \mathrm{OCHCH}_{2}$, anti isomer), 3.38$3.28\left(0.34 \mathrm{H}, \mathrm{m}, \mathrm{OCH}_{2} \mathrm{CH}_{2}\right.$, syn isomer $), 3.18(0.83 \mathrm{H}, \mathrm{d}, J=4.0 \mathrm{~Hz}, \mathrm{OH}$, anti isomer $), 2.80$ $(0.17 \mathrm{H}, \mathrm{d}, J=4.4 \mathrm{~Hz}, \mathrm{OH}$, syn isomer $), 2.44-2.35\left(0.83 \mathrm{H}, \mathrm{m}, \mathrm{OCH}_{2} \mathrm{CH}\right.$, anti isomer $), 2.28$ $2.25\left(0.17 \mathrm{H}, \mathrm{m}, \mathrm{OCH}_{2} \mathrm{CH}\right.$, syn isomer), 2.21-2.10 (1H, m, $\left.\mathrm{OCH}_{2} \mathrm{CH}\right) ;{ }^{13} \mathrm{C} \mathrm{NMR} \mathrm{(100} \mathrm{MHz,}$ $\left.\mathrm{CDCl}_{3}\right) \delta 138.23,137.44,137.36,129.01,128.87,128.57,128.48,128.29,127.67,127.54$, $126.72,126.02,90.57,89.99,75.50,74.14,73.22,73.09,65.97,65.70,30.71,28.20$, four signals of syn isomer were missing; IR (liquid film) 3423, 1653, 1549, 1454, 1367, 1094, 768, 741, $700 \mathrm{~cm}^{-1}$; HRMS (ESI-TOF) calcd for $\mathrm{C}_{17} \mathrm{H}_{19} \mathrm{NNaO}_{4}: 324.1206\left([\mathrm{M}+\mathrm{Na}]^{+}\right)$. Found: $324.1208\left([\mathrm{M}+\mathrm{Na}]^{+}\right)$; HPLC conditions DAICEL Chiralpak AS, hexane/2-propanol = 10:1, flow rate $=0.5 \mathrm{~mL} / \mathrm{min}$, retention time; $27.2 \mathrm{~min}, 29.7 \mathrm{~min}$ (anti); $33.0 \mathrm{~min}, 43.0 \mathrm{~min}$ (syn); [70\%, anti/syn $=87: 13,91 \%$ ee (anti isomer), $35 \%$ ee ( $s y n$ isomer)]. 
catalytic amount of Raney nickel under a hydrogen atmosphere in methanol at room temperature followed by the reaction with di-tert-butyl dicarbonate (3 equiv) and a catalytic amount of DMAP (20 mol\%) gave 2-N-Boc-amino-1-phenyl-1-propanol (4). ${ }^{4}$ The comparison of its HPLC retention time with that of $(1 R, 2 S)-2-N$-Boc-amino-1-phenyl-1propanol independently prepared from commercially available $(1 R, 2 S)-(-)$-norephedrine confirmed that the absolute configuration of the nitroaldol product 3a was $(1 R, 2 S)$. 4: HPLC conditions: DAICEL Chiralpak AS, hexane/ethanol $=10: 1$, flow rate $=0.5 \mathrm{~mL} / \mathrm{min}$, retention time; $9.5 \mathrm{~min}$ [minor isomer $(1 S, 2 R)$ ], $10.0 \mathrm{~min}$ [major isomer $(1 R, 2 S)$ ].

NMR Study. The ${ }^{19}$ F-NMR spectra of a solution of chiral quaternary ammonium bifluoride $1 \mathbf{b}(80 \mathrm{mg}, 0.05 \mathrm{mmol})$ in THF- $d_{8}(600 \mu \mathrm{L})$ was measured at $-78{ }^{\circ} \mathrm{C}$, which showed broad resonance centered at $\delta-149.8 \mathrm{ppm}$. With the addition of trimethylsilyl nitronate $(2.4$ equiv, $20 \mu \mathrm{L}, 0.12 \mathrm{mmol}$ ) at $-78^{\circ} \mathrm{C}$, the signal of trimethylsilyl fluoride appeared at $\delta-157.61 \mathrm{ppm}$, supporting generation of the corresponding chiral ammonium nitronate. [Chemical shifts are reported from fluorobenzene resonance $(-113.0 \mathrm{ppm})$ as the external standard.]

\section{References}

(1) (a) Ando, A.; Miura. T.; Tatematsu, T.; Shioiri, T. Tetrahedron Lett. 1993, 34, 1507. (b) Shioiri, T.; Bohsako, A.; Ando, A. Heterocycles 1996, 42, 93. (c) Horikawa, M; BuschPetersen, J.; Corey, E. J. Tetrahedron Lett. 1999, 40, 3843.

(2) Seebach, D.; Beck, A. K.; Mukhopadhyay, T; Thomas, E. Helv. Chim. Acta. 1982, 65, 1101.

(3) The references for nitroaldol adducts; (3a) Sasai, H.; Hiroi, M.; Yamada.Y. M. A.; Shibasaki, M. Tetrahedron Lett. 1997, 38, 6031. (3b) Palacios, F.; Aparicio, D.; De los Santos, J. M.; Baceiredo, A.; Bertrand, G. Tetrahedron 2000, 56, 663. (3c) and (3d) (a) Kumar, H. M. S.; Reddy, B. V. S.; Yadav, J. S. Chem. Lett. 1998, 657. (b) Marao, I.; Cossío, F. P. Tetrahedron Lett. 1997, 38, 6461. (3e) See ref. 2.

(4) (a) Christensen, C.; Juhl, K.; Hazell, R. G.; Jørgensen, K. A. J. Org. Chem. 2002, 67, 4875. (b) Besse, P.; Veschambre, H. J. Org. Chem. 1994, 59, 8288.

\section{${ }^{1} \mathrm{H}$ and ${ }^{13} \mathrm{C}$ NMRs of New Compounds.}



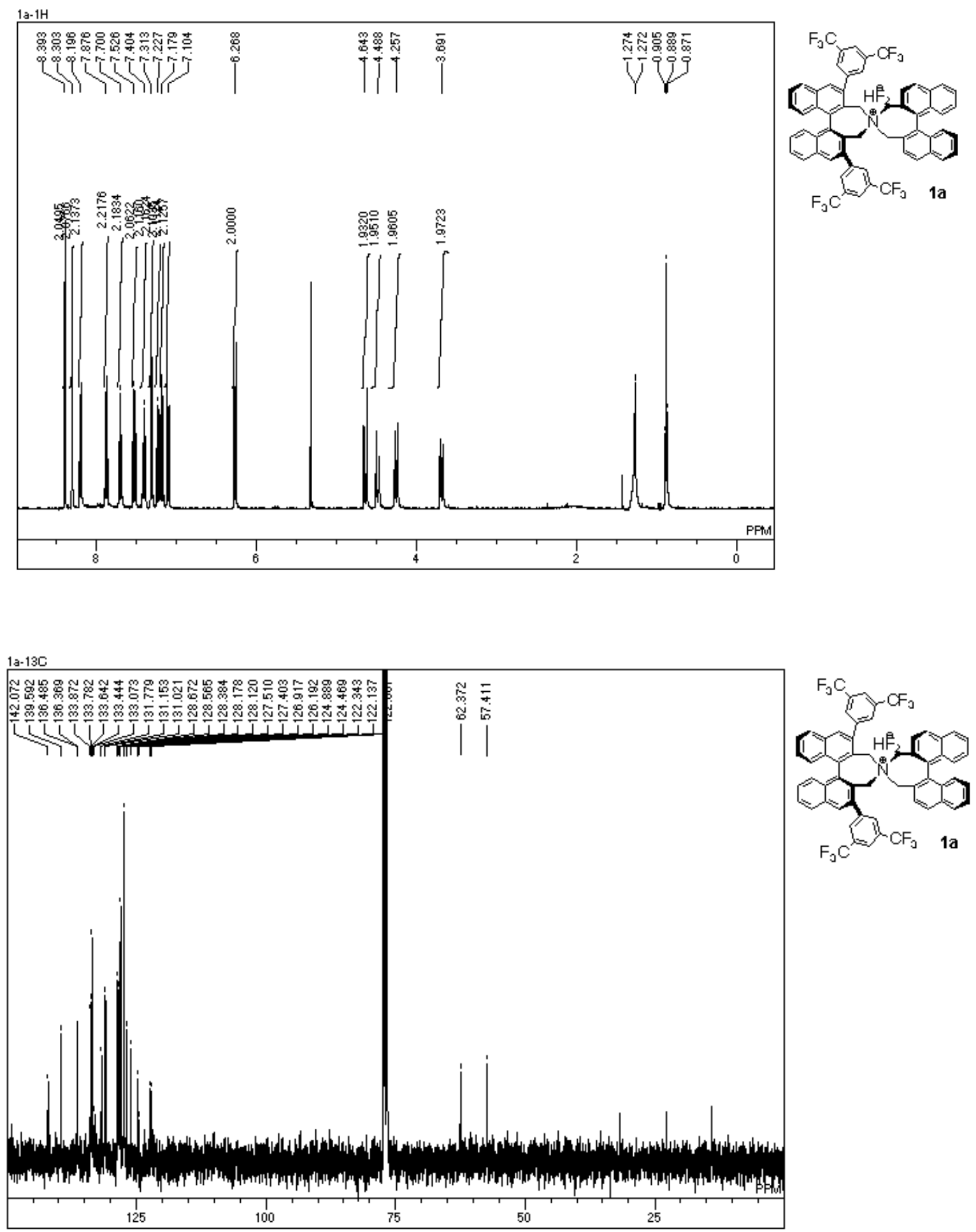

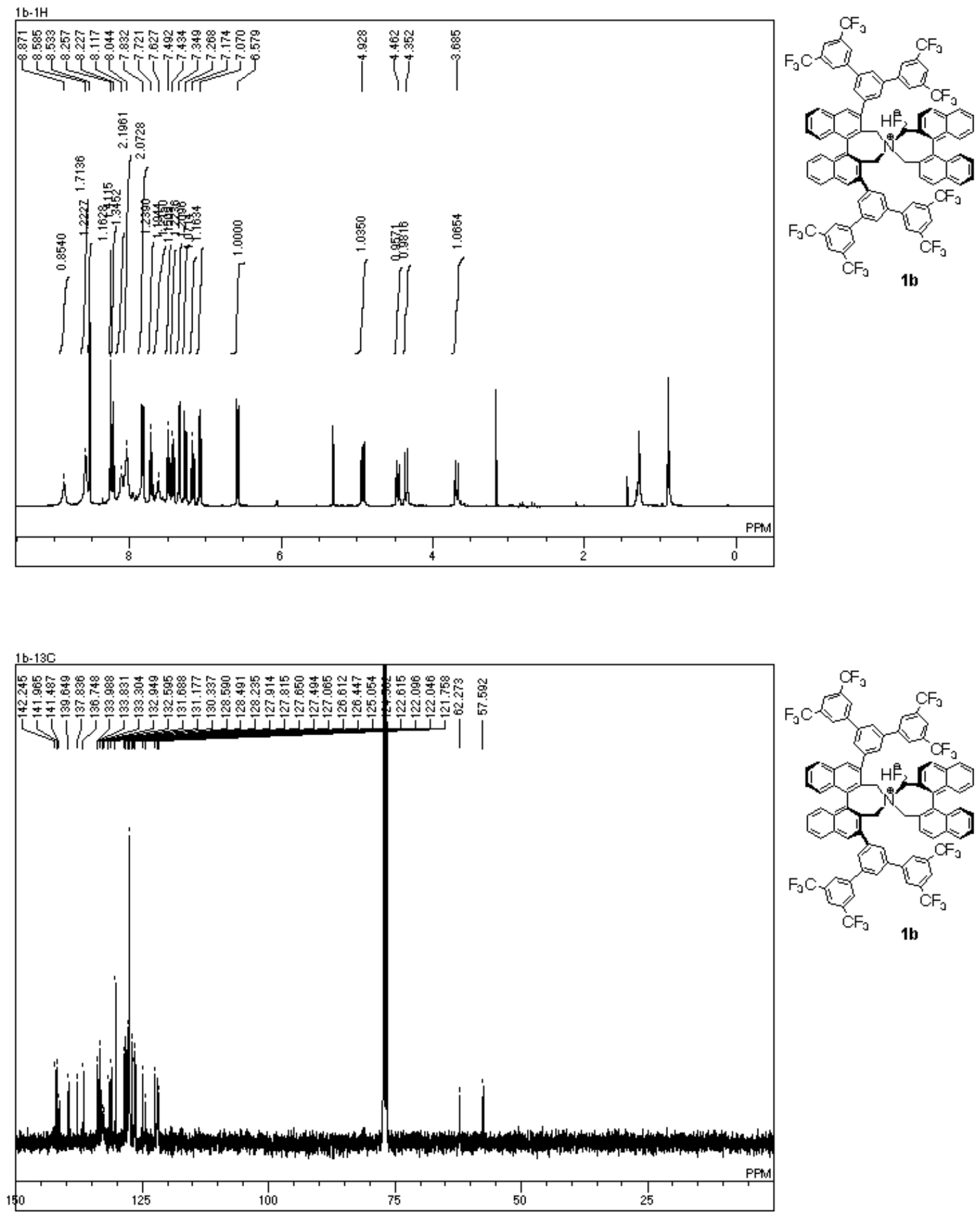

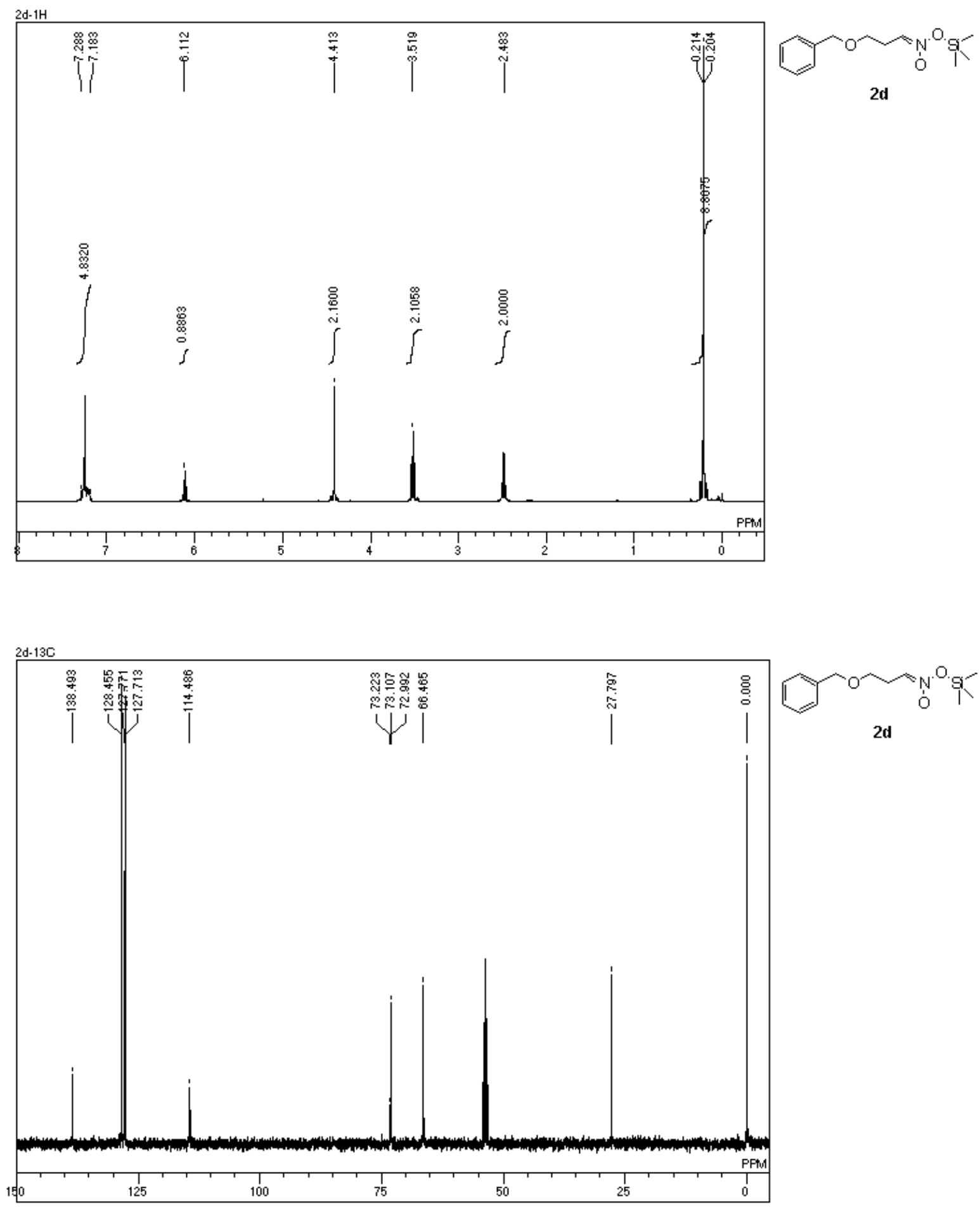

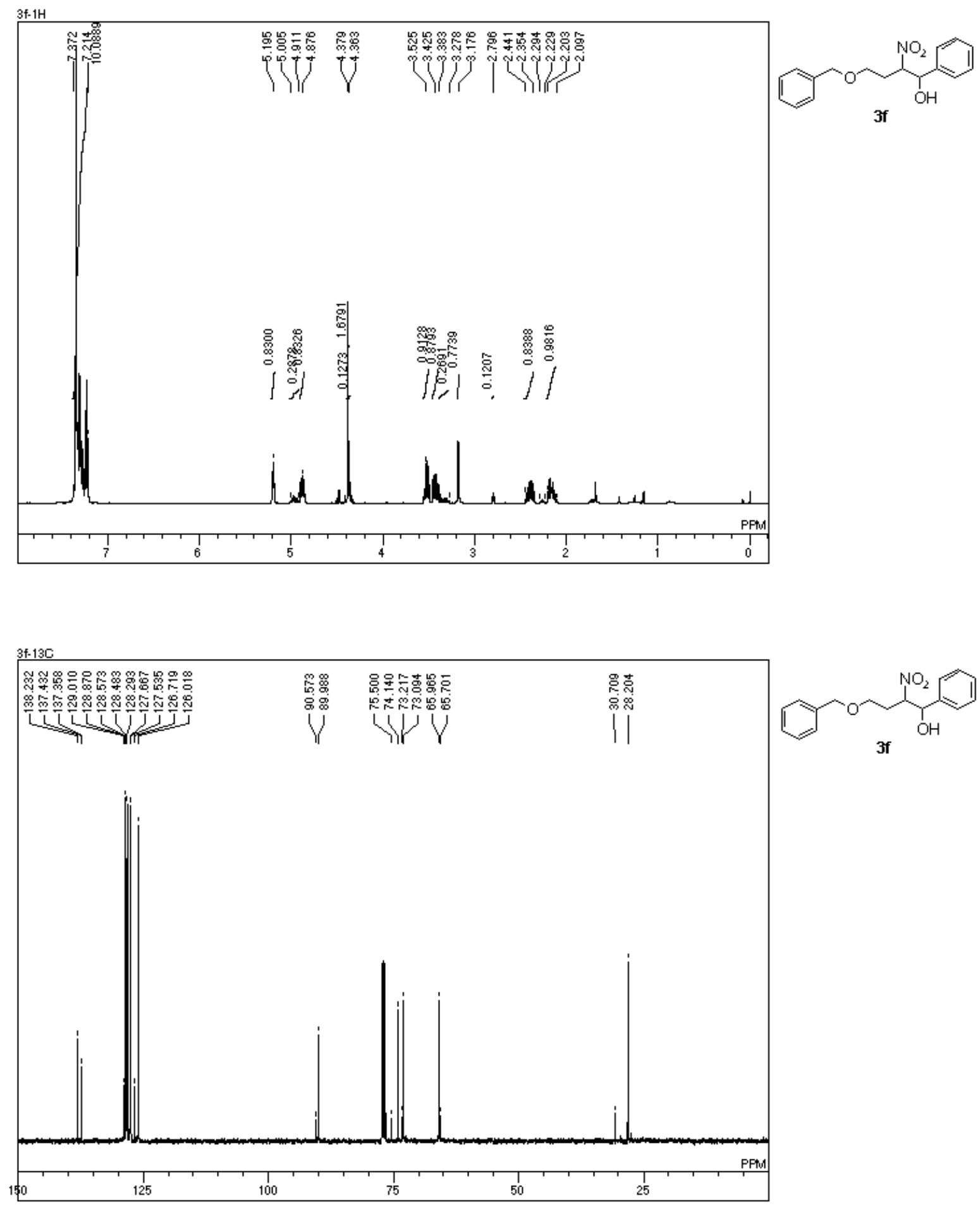